\title{
INTERFERENZE SINTATTICHE DI ORIGINE ROMANZA NELLE PARLATE SLOVENE OCCIDENTALI: LA STRUTTURAZIONE DEL SiNTAGMA AgGeTTIVALE, DELla FRASE, DEL PERIOdO
}

1. Il Festeggiato si è occupato, a più riprese, delle interferenze linguistiche tra il mondo slavo e quello romanzo; ha dedicato, e dedica tutt'ora, una buona parte dell'energia e dell'attività scientifica all'istroromanzo, il quale istroromanzo sta in opposizione diglossica, quale registro basso, rispetto al serbocroato, da una parte, e all'italiano, e magari alla koinè veneta, dall'altra. Il Tekavčić ci ha offerto delle analisi interessanti ed esaustive, delle quali ha beneficiato anche questa rivista, nel campo semantico e lessicale. Non ha trascurato nemmeno problemi concernenti le interferenze sintattiche, e questo fin dalle sue ricerche sulla parlata di Dignano. Il problema della consecutio temporum, ad esempio.

Voglio sperare pertanto di non aver fatto una scelta sgradita proponendomi come tèma per la Miscellanea a suo onore la presentazione di una situazione analoga, benché a rovescio. Prendo in esame, infatti, le influenze romanze, friulane, più che altro, che riscontriamo nelle parlate slovene occidentali, in Resia, nella valle del Torre e nelle alte valli del Natisone; nella provincia di Udine, cioè. La situazione in cui versa lo sloveno di questo territorio è abbastanza analoga a quella dell'istroromanzo: anche lo sloveno ha come registro alto, oltre allo sloveno letterario, l'italiano standard. Aggiungiamo che il suo valore sociolinguistico, vale a dire nella vita pubblica, è scarso, giacché non gode, al pari del friulano, di nessuno status ufficiale. Poi, contrariamente alla situazione a Trieste o a Gorizia, non ci sono, e non ci sono state mai, scuole pubbliche statali in sloveno.

2. Il nostro interesse va verso i fenomeni sintattici dove crediamo di vedere influssi romanzi. Limiteremo la nostra ricerca al problema della strutturazione degli elementi costitutivi del sintagma aggettivale, della frase semplice e del periodo. Delimitata così l'area della nostra ricerca dal punto di vista geografico e contenutistico, conviene precisare che le fonti della nostra conoscenza sono inchieste dirette sul luogo, a cominciare da quelle di Baudouin de Courtenay. Materiale prezioso ci è offerto, inoltre, dalla tradizione orale confluita in raccolte etnologiche, fiabe e leggende in particolare. ${ }^{1}$

1 J. Baudouin de Courtenay, Materialien zur südslavischen Dialektologie und Ethnographie, I-III (Mat.), rispettivamente St. Petersburg, 1895, 1904, 1913, e Materiali per la dialettologia e l'etnografia slava meridionale, IV, Trieste-Trst 1988; T. Logar, Slovenska narečja (Dial.), Ljubljana 1975;

M. Matičetov, 3 lesičice gotrce /3 comari volpicelle/, Trst-Ljubljana 1987. 
3. La strutturazione del sintagma aggettivale non offre motivi di interesse particolare. Si parte dalla norma valida per lo sloveno: l'aggettivo come attributo, quale che sia il suo valore, connotativo o denotativo, precede il sostantivo. Solo le esigenze di stile possono far cambiare l'ordine fisso, e ancora in un numero molto limitato di casi. Di conseguenza, quando nel nostro materiale scorgiamo un aggettivo che segua al sostantivo, senza che si possa constatare una spinta stilistica, siamo inclini a vedervi una imitazione del modulo italiano o friulano, un calco sintattico, cioè. Esempi, non molti, ma significativi, se ne trovano già nelle inchieste del Baudouin: Trava zalaná; arbol zalèn 'erba verde, albero verde' (Mat. I, 1098); Mir bil. Lapiš černjel. Jisi lapiš rus 'muro bianco, matita rossa, questa matita gialla' (ibid., 1251 e 1252); Ja znan kanconet slovejskih dosti an dosti 'conosco davvero molti canti sloveni' (Mat. II, 66); Se majò potpisate tapo dan kontrat semplic 'devono /i neosposi/ sottoscrivere un contratto semplice' (ibid., 209); Mèšta se naredi, se skuha voz moke sjerkove 'la polenta si fa, si cucina con la farina di granoturco' (ibid. 247).

Passi analoghi si trovano anche nei testi più vicini ai nostri tempi. Nei racconti animaleschi incontriamo Den lipi kafe črne 'un bel caffè nero'. Dalle fiabe possiamo citare il nome di Kapučeto roso che è un prestito per lo sloveno Rdeča kapica; però, la disposizione romanza degli elementi risulta chiara nel sintagma che è nome comune: N'e mama ju je klicala Kapučeto roso, zatou $k$ je miela simper na lave nu kapučecu čerńelu 'la mama la chiamava Cappuccetto rosso perché portava sul capo sempre un cappuccio rosso' (Dial., località Subid).

Gli esempi addotti mostrano una disposizione degli elementi del sintagma tipicamente romanza. C'è da osservare che in tutti i passi l'aggettivo ha un chiaro valore denotativo; non sorprende perciò che siano interessati i nomi dei colori.

4. $\mathrm{Ci}$ attrae, nel presente contributo, in maniera particolare la strutturazione della frase semplice. E' proprio qui che vediamo una dissonanza significativa tra la strutturazione della frase, vale a dire, tra il posto assegnato a singoli elementi, come li troviamo nel materiale esaminato, e la norma, valida per lo sloveno standard. Constatiamo infatti un'insolita posizione dei clitici i quali, in sloveno, seguono al primo elemento della frase: ne segue che la frase cambia d'aspetto se il primo posto viene occupato da un altro elemento e non dal soggetto che anche in sloveno, di regola, occupa il primo posto. ${ }^{2}$ Cosi in italiano come in friulano la struttura della frase semplice, dal modulo S-V-O, rimane intatta, anche se introdotta da un altro elemento, ad esempio da una locuzione avverbiale. Tale struttura, nello sloveno occidentale, va dunque considerata un calco sintattico dal romanzo. Il fenomeno era stato già trattato, in questa rivista, sulla base dei compiti scritti della scolaresca slovena in una scuola slovena di Trieste. ${ }^{3}$ L'esame del nostro materiale, in parte le in-

2 Dell'ordine degli elementi in sloveno avevano discusso già alcuni linguisti sloveni nella seconda metà del secolo scorso. Per la questione si veda J. Toporišič, Nova slovenska skladnja /La nuova sintassi slovena/, Ljubljana 1983, pp. 204 ss.

3 Cf. F. Ferluga, Problemi di interferenza linguistica: su un errore di sintassi slovena degli studenti sloveni bilingui, Linguistica XXII (1982), pp. 171-189. 
chieste del Baudouin effettuate cento anni fa, in parte materiale etnologico, raccolto nel secondo dopoguerra (ma è ovvio che l'esploratore si rivolge quasi sempre alla gente di una certa età) può dunque servire da controprova, perché si tratta di territorio e di tempo dell'inchiesta distinti. La constatazione dell'esistenza del fenomeno anche in epoche precedenti prova, infatti, che il fenomeno non è attribuibile solo alla lingua dei giovani, all'influsso della scuola o della lettura dei giornali italiani; deve essere di antica data, conseguenza della simbiosi secolare tra le due etnie.

La maggior parte dei casi mostra il cambio del posto del clitico quando la frase incomincia con una locuzione avverbiale di tempo o di luogo: U Nedejo uzhilo Christjanske je bilu tau Parochj 'domenica scorsa il catechismo ebbe luogo in parrocchia' (Mat. III, 57); Prit annikoi Christus je térpel martro, anù od smerti ustal, Apostolovi so bili teliku toshni anù strashni, da koi je bil Iesus Christus jet, na mestu ga vibranit, ni su ussi zhistu ubishali 'prima che Cristo patisse il martirio e risorgesse, gli apostoli erano così tristi e spaventati che, quando Gesù Cristo fu preso, anziché difenderlo, tutti fuggirono' (ibid., 97); Ankrat na lesica je bla ušafala adno kakuoš 'una volta una volpe aveva preso una gallina'; Antadá lesica an vuk sta šla nepri an sta gvorila met sabo 'e allora la volpe e il lupo continuarono per la strada e chiacchierarono tra di loro' (Mat. IV, 9); Dol po poti Toninac je sreču dnu petjarcu 'per il cammino, Toni ha incontrato una mendicante' (Dial., località Sarženta).

In tutti questi casi la norma sintattica dello sloveno richiederebbe la posizione del clitico, sia un ausiliare che una forma pronominale atona, immediatamente dietro il primo elemento. La strutturazione alla romanza non è, nei testi esaminati, esclusiva (si veda nel Mat. III, 97 koi je bil lesus Christus jet), è però frequente, senza essere predominante.

Troviamo un procedimento analogo anche in una principale quando è una subordinata che precede: Sin, ko je čul, da oća se mimbra an je vilizal u mlin 'il figlio quando udi che il padre si lamentava, entrò nel mulino' (Mat. I. 23); Kar sierak je biu zamliet, mlinar mu je oduzeu ńeha miercu 'quando il granoturco fu macınato, il mugnaio gli tolse la quantità che a lui, mugnaio, spettava' (Dial., Sarženta). Infine, alle volte si trova la stessa conservazione dell'ordine degli elementi, S-V-O, anche nelle subordinate oggettive, quando precede la principale: Berič (finanče) su pensale, de vi ste ot papeša špijon 'i dazieri pensarono che Lei fosse una spia papalina' (Mat. II, 18); Medvet je mislil, da človek je marou 'l'orso credeva che l'uomo fosse morto' (ibid., 104).

Strutturazione non dissimile si trova nel friulano; citiamo dallo Sgorlon: $D$ ' unviâr la mê vite 'e coreve dentri la cjase di piere 'd'inverno, la mia vita scorreva dentro della casa di pietra'; Ma par furtune jê 'e jere pronte a difindimi 'per fortuna lei era pronta a difendermi'; Di quanche il Nono al jere lât cun Diu, 'da quando il nonno se n'era andato' (Il dolfin, I, Lis fiestis). ${ }^{4}$

4 Carlo Sgorlon, Il dolfin, Udin 1982. 
5. Per contro, la strutturazione del periodo non presenta problemi di rilievo, vale a dire, non ci sono dissonanze nella strutturazione del periodo tra le parlate slovene che stiamo esaminando e lo sloveno letterario. La disposizione degli elementi del periodo, delle singole proposizioni, cioè, è regolata in un modo analogo nello sloveno e nelle lingue romanze esaminate. In sintesi, precedono la principale le subordinate che in qualche maniera condizionano o preannunciano la realizzazione dell'azione espressa nella principale; così, la maggior parte delle temporali, le causali, le condizionali. Le concessive, in una parlata spontanea, quasi quasi non ci sono. Seguono, invece, le oggettive, le finali, le consecutive, le modali e comparative, mentre le più libere, quanto alla collocazione, sembrano le subordinate soggettive. Per ovvie ragioni, alla rispettiva antecedente seguono le attributive, le quali, a rigore, non fanno parte del periodo.

Citiamo per le subordinate preposte, oltre agli esempi già citati: Ko $n$ se ustavil tana dureh, štrija je rakla 'quando si fermò alla porta, la strega disse'; Koj te drugi ni piršal, ti boš mažal tit ti 'siccome non è tornato quell'altro, devi andartene tu'; be a mel jito vodo, da am bej tel se ozdravit'/il padre aveva fatto un sogno che/ se avesse tale acqua, potrebbe guarire'. E per le subordinate posposte: Anu se pumimbral, da om bej tel rade jitit po to vodo za naga oćo 'si lamentò che volentieri sarebbe andato lui a cercar l'acqua per il babbo'; Ja con ti naredet den $\mathrm{kruh}$, den hlep kruha, da ti boš mel za tuo pot, na vijač 'ti farò un pane, perché tu lo prenda per il viaggio'; $D$ an ga mej naprit utvupit nutu to viliko vodo, da an ga ni vidi već 'che deve lui, mugnaio, affogarlo perché egli, il padre, non lo veda più'; Ja gren jo po za miga oćo ke je bolan 'vado a cercare /l'acqua/ per il mio babbo che è malato'. (Dal racconto del re che aveva tre figli, Mat. $1,1-27$ ).

Anche qui conviene mettere a confronto alcuni passi dalla stessa opera letteraria dello Sgorlon: Quanch'a scomenčavin lis primis neveadis in te valade, in cjase mê si podevin cucá i prins segnâi des Fiestis 'quando cominciavano le prime nevate nella valle, si scoprivano a casa mia i primi segnali delle Feste' contro a Jo lis nulivi tal ajar come ch'a fossin un odôr 'io le sentivo nell'aria, come se fossero un odore'; $E$ ' viveve simpri cul cûr in man parvìe de pôre ch'o mi cjapàs alc par colpe dal frêt 'lei viveva sempre col cuore in mano, a causa della paura che io prendessi qualcosa per colpa del freddo'; E' jere convinčude che jê 'e jere l'uniche a veglâ di e gnot parsore di me 'era convinta che solo lei potesse vegliare sopra di me giorno e notte'; Tal prât mi sintivi esponût, senze difese, come se lis robis plui strambis e mai sintudis a dî $a$ ' podessin sucedimi di colp 'sul prato mi sentivo esposto, senza difesa, come se cose più strane e mai sentite raccontare potessero succedermi'.

La strutturazione analoga del periodo non va dunque spiegata con una ipotetica influenza linguistica. La concordanza invita a considerare il fenomeno universale $o$, almeno, noto a più lingue. La strutturazione è condizionata da esigenze psicologiche. 
SKLADENJSKA VPLIVANJA ROMANSKEGA IZVORA V ZAHODNIH SLOVENSKIH GOVORIH: GRADNJA PRIDEVNIŠKE SINTAGME, STAVKA, POVEDI

Za gradnjo nekaterih skladenjskih enot se skuša ugotoviti, ali se v zahodnih slovenskih govorih (Rezija, dolina Tera, doline gornje Nadiže) kaže romanski jezikovni vpliv. Pridevnik kot prilastek ima v slovenščini stalno mesto pred samostalnikom, ki ga določa; kadar torej na slovenskem zahodu ni tako ("kava črna“), imamo ta pojav za skladenjski kalk po furlanščini ali italijanščini, kjer prilastek denotativne, razločevalne vrednosti zmeraj sledi. Ravnotako ugotavljamo romanski vpliv pri gradnji stavka: naslonka zaseda v slovenščini drugo mesto, tik za prvo polnopomensko besedo; potemtakem se besedni red spremeni, če postavimo na čelo stavka kako prislovno določilo. V romanskih jezikih pa na besedni red dodajanje kakega elementa nič ne vpliva. Ce torej najdemo podobno gradnjo stavka v slovenskih zahodnih govorih, smemo pomisliti na romanski skladenjski vpliv. Ne moremo pa govoriti o tujem jezikovnem vplivanju pri gradnji povedi: tu ni razlike med slovenščino in obema romanskima jezikoma, ki sta $\mathrm{z}$ njo $\mathrm{v}$ neposrednem stiku: urejanje členov povedi pogojujejo psihološki razlogi. 